\title{
Confiabilidad y validación de un modelo de formulación clínica conductual ${ }^{*}$
}

\author{
Reliability and Validation of a Behavioral Model \\ of Clinical Behavioral Formulation
}

Recibido: marzo 19 de 2010 | Revisado: junio 11 de 2010 | Aceptado: julio 26 de 2010

\author{
AMANDA M. MuÑOZ-MARTINEZ ** \\ MÓNICA MA. NOVOA-GÓMEZ \\ Grupo de Investigación en Psicología y Salud, \\ Pontificia Universidad Javeriana, Bogotá, Colombia
}

SICI: 1657-9267(201108)10:2<501:CVMFCC > 2.0.TX;2-K

Para citar este artículo. Muñoz, M. A. \& NovoaGómez, M. M. (2011). Confiabilidad y validación de un modelo de formulación clínica conductual. Universitas Psychologica, 10 (2), 501-519.

* Artículo de investigación. Las autoras agradecen a los colegas que trabajaron en la ejecución del convenio de Jóvenes investigadores e Innovadores de Colciencias y la Pontificia Universidad Javeriana, número 00003303.

** Facultad de Psicología, Cra. 5 \# 39-00 piso 2. Correos electrónicos: amanda.munoz@javeriana.edu. co;mmnovoa@javeriana.edu.co

Research-ID: Muñoz-Martinez, A. M., E-14282011; Novoa-Gómez, M. M., E-1869-2011

\section{RES U MEN}

El objetivo de la presente investigación fue determinar la confiabilidad y la validez de contenido y predictiva de un modelo de formulación, desarrollado desde una perspectiva conductual. Para ello, se utilizó un diseño de investigación mixto que integró niveles de análisis descriptivos y experimentales de caso único A-B-con seguimiento. Se analizó la fiabilidad de las siguientes categorías descriptivas y explicativas: (a) problemas, (b) predisponentes, (c) precipitantes, (d) adquisición y (e) mecanismos inferidos, en formulaciones hechas por terapeutas entre el años 2005 y 2008, y por otros psicólogos con el modelo derivado del presente estudio. Con respecto a la validez, jueces expertos determinaron que el modelo tenía validez de contenido, y a través del uso de éste en tres estudios de caso, se determinó que tenía validez predictiva. Se discute la importancia de ampliar la investigación con otras poblaciones y establecer la validez concurrente y clínica del modelo. Palabras clave autores

Evaluación conductual (Sc05671), modelo comportamental (Sc05690), validez (Sc52280), confiabilidad (Sc11230).

Palabras clave descriptores

Ciencias del comportamiento, investigación psicológica, psicología clínica, análisis de datos.

\section{A B S T R A C T}

The aim of this investigation was determined the reliability and content and predictive validity of a clinical case formulation, developed from a behavioral perspective. It was used a mixed design that integrated levels of descriptive analysis and unique case study, A-B with follow. In this study was established the reliability of follow descriptive and explanatory categories: (a) problem description, (b) predisposing, (c) precipitating, (d) acquisition and (e) inferred mechanism (maintenance), the analysis was did with cases attended between 2005 and 2008 and persons that was formulated with the model derived of the current investigation. Regard to validity, expert judges considered that the model had content validity. The predictive validity was established across application of model to three case studies. Discussion shows the importance to extend the investigation with the model in other population and determinate the interformulator and clinical validity of these.

Key words authors

Behavior Assessment (Sc 05671), Behavioral Model (Sc 05690), Validity (Sc52280), Reliability (Sc11230).

Key words plus

Behavioural Sciences, Psychological Research, Clinical Psychology, Data Analysis. 
Dentro de la perspectiva comportamental se han diseñado modelos de formulación a partir de las diferentes posturas que hacen parte del paradigma, y aunque comparten algunos elementos entre sí, se observan diferencias importantes entre ellos, de forma que mientras algunos parten de posturas ídiográfico-inductivas (Caycedo, Ballesteros \& Novoa, 2008; Haynes, S. \& O’Brien, 2000; Pachón \& Novoa-Gómez, 2009) otros lo hacen desde una visión nomotética-deductiva (Nezu, Nezu \& Lombardo, 2004). Estas diferencias implican ciertas consecuencias en relación con la forma en la que se conceptualizan los casos y así mismo en los resultados finales de las intervenciones terapéuticas (Muñoz \& Novoa, 2009, 2010).

Desde hace unos años, los estudios relacionados con la formulación de caso han incrementado de manera notoria (Bergner, 1998; Castro \& Angel, 1998; Caycedo et al., 2008; Eells, 2001, 2007; Eells, Kendjelic \& Lucas, 1998; Graña, 2005; Hayes \& Hass, 1988; Haynes, Leisen \& Blaine, 1997; Nezu et al., 2004; Pachón \& Novoa-Gómez, 2009), reconociendo el valor de su uso tanto psicólogos como profesionales en otras áreas de la salud (Sim, Peng $\&$ Bateman, 2005). A pesar de las diferencias que subyacen a cada una de las perspectivas que hacen uso de ella, se puede observar que los autores la definen en general como una metodología que le permite al psicoterapeuta ordenar la información derivada de la evaluación, para describir y explicar las problemáticas y con base en ello elaborar un plan de intervención que resulte pertinente para las personas que asisten a consulta, lo que ayuda a incrementar la posibilidad de obtener resultados terapéuticos exitosos (Caycedo et al., 2008) y facilitar entrenamiento y ejercicio profesional de nuevos terapeutas (Kendjelic \& Eells, 2007).

No obstante, el uso de la formulación no es suficiente para garantizar que los terapeutas deriven intervenciones del análisis de las relaciones que existen entre los eventos del contexto actual, la historia del individuo y el comportamiento problema, pues se ha observado que en muchas ocasiones ellas se llevan a cabo con base en el juicio clínico intuitivo del profesional y no en las hipótesis establecidas desde el modelo, contrario a lo que se esperaría de las metodologías enmarcadas dentro de la perspectiva conductual (Iwata \& Worsdell, 2005; Kahneman \& Klein, 2009).

Con el fin de establecer los factores involucrados en ello, junto a la evaluación de la utilidad y pertinencia de las formulaciones, se han hecho estudios en los que se ha encontrado que la mayoría de los modelos tienen principalmente tres problemas. El primero se refiere a dificultades de tipo conceptual, pues se ha visto que tras el análisis que se hace de la información no es posible identificar si existe coherencia entre las hipótesis derivadas de éste y los supuestos teóricos desde una perspectiva conceptual determinada, especialmente en lo relacionado con los niveles de análisis que se plantean para dar explicación a la conducta (Rodríguez \& Vanegas, 2010). El segundo se relaciona con los supuestos epistemológicos del que parten sus afirmaciones, pues éstas tienden a centrarse, de manera especial, en una visión metodológica y práctica sin hacer explícitas las bases teóricas de las que se parte (Rodríguez \& Vanegas, 2010), lo que se encuentra en contraposición con los supuestos de base en el análisis del comportamiento, los cuales plantean que las explicaciones deben darse en relación con lo encontrado a través de la investigación tanto básica como aplicada y las teorías derivadas de dichos hallazgos (Arias, Fernández \& Perona, 2000). Por último, se ha observado que éstas tienen dificultades, para mostrar la utilidad de la información recolectada de acuerdo con lo que exige el modelo para la práctica clínica (Rodríguez $\&$ Vanegas, 2010), siendo en ocasiones poco parsimoniosas (Caycedo et al., 2008) y facilitando la elaboración de explicaciones, sin tener en cuenta las descripciones hechas de los casos en el modelo, dada la poca claridad que se tiene respecto a lo que es pertinente o no para ellas (Rodríguez \& Vanegas, 2010).

Debido a esas dificultades, se ha planteado, desde una perspectiva comportamental, la importancia de diseñar modelos de formulación con base en una visión idiográfica (Eells, 2001), que se ajuste a los postulados del análisis conductual, en los que se plantea la importancia de establecer las relaciones entre la información recolectada 
y los supuestos teóricos de esta perspectiva, los cuales se han derivado de la investigación básica y aplicada (Arias et al., 2000). Sumado a ello se ha enfatizado en la importancia de establecer la confiabilidad (Eells et al., 1998) y la validez (Mumma \& Mooney, 2007; Mumma \& Smith, 2001) de las categorías utilizadas en ellas, pues, como se ha visto, no es suficiente con ajustar los datos a ellas, sino que es importante conocer a priori como lo plantean Carey, Flasher, Maisto y Turkat (1984) los elementos que resultan útiles para dar cuenta de la problemática, y posteriormente fundamentar o derivar los elementos explicativos de tales análisis.

Con este fin, el Grupo de Investigación en Psicología y Salud de la Pontifica Universidad Javeriana ha realizado una serie de investigaciones con el propósito de subsanar parte de esos problemas, y obtener un modelo válido y confiable desde una perspectiva idiográfica, con base en los postulados del análisis del comportamiento. El primero de los estudios dirigidos a la elaboración del modelo de formulación clínica, fue desarrollado por Caycedo et al. (2008), en el que se estableció la formulación de caso con base en el modelo de bienestar, de éste se derivaron, como principales elementos explicativos, la predicción y el control, los cuales implicaban la comprensión de las relaciones de interdependencia, entre el comportamiento y su contexto, a través de la identificación de las relaciones funcionales que se daban entre estos dos; sin embargo, tras el proceso de validación de contenido se encontró que estas resultaban ser generales y podían llegar a conclusiones reduccionistas de los casos. Debido a ello, se planteó la importancia de establecer categorías más específicas, con el fin de dar cuenta de las problemáticas, y así evitar que de ella se derivaran intervenciones que no tuviesen en cuenta las características particulares de los casos, olvidando el carácter idiográfico de esta metodología.

Para contrarrestar las limitaciones y mejorar la predicción de éxito terapéutico, Pachón y NovoaGómez (2009) modificaron las categorías utilizadas en la investigación previa y propusieron, como posibles elementos explicativos, el autocontrol, la regulación verbal de la conducta, los repertorios para modificar el ambiente y los repertorios de autoconocimiento. Los resultados de dicha investigación mostraron que la forma de presentar la información en la formulación clínica, les permitía a los terapeutas tener información suficiente acerca de los casos, de tal forma que, tanto para los psicólogos novatos como para los expertos, resultaba útil. Sin embargo, los resultados de la validación de contenido mostraron que las categorías utilizadas para explicar el comportamiento, tenían un carácter descriptivo más no explicativo. Además, presentaba algunas dificultades con respecto a su uso por parte de los psicólogos pertenecientes a diferentes posturas dentro del modelo conductual, dado que algunas de las categorías utilizadas se relacionaban directamente con una perspectiva específica, como por ejemplo, la regulación verbal del comportamiento que parte de un visión no mediacional dentro del paradigma.

Debido a lo anterior, la presente investigación se desarrolla con el fin de trabajar las limitaciones encontradas en los anteriores estudios, por lo que se hace uso de categorías que parten del análisis de casos dentro de la perspectiva conductual, siguiendo el Método de Formulación de Caso de Codificación de Contenido (CFCCM, por sus siglas en ingles) desarrollado por Eells et al. (1998). Las categorías trabajadas por dichos autores son: la descripción del problema, los factores de predisposición, los factores desencadenantes y los mecanismos inferidos.

La descripción del problema implica la identificación de aquellos elementos que pueden tener algún tipo de relevancia clínica y de los elementos relacionados con su presentación, estos permiten al terapeuta determinar el problema, aunque éste no sea obvio para el consultante (Eells et al., 1998). Mientras los factores de predisposición hacen referencia a eventos ocurridos en la vida del individuo, que incrementan la vulnerabilidad de desarrollar determinados problemas (Eells et al., 1998; Graña, 2005; Nezu et al., 2004). Con respecto a los factores precipitantes, estos hacen referencia a eventos que exacerban o sirven como "disparadores" para la aparición de los problemas (Eells et al., 1998). Por último, los mecanismos 
inferidos implican los elementos que relacionan la información contenida en las otras categorías, y dan explicación del mantenimiento del problema en la actualidad, organizando la información para conceptualizar el caso (Eells et al., 1998), estos son denominados en modelo conductual como factores de mantenimiento (Graña, 2005). Sumado a ellos, dentro del paradigma, también se hace uso de una última categoría para explicar el comportamiento, los factores de adquisición, que hacen referencia a la forma en que se relacionan las variables en la consolidación del problema y su curso (Eells, 2007; Graña, 2005); cabe señalar que, a pesar del uso que tiene por parte de los terapeutas para conceptualizar los casos, se han encontrado ciertas dificultades por parte de ellos para diferenciarla de otros aspectos y comprender su función dentro de la formulación clínica.

Con el fin de establecer la función de las categorías mencionadas previamente en la compresión de los casos y su utilidad, el objetivo de la presente investigación fue determinar la confiabilidad de las categorías explicativas utilizadas en las formulaciones clínicas enmarcadas en el modelo comportamental, y la fiabilidad y validez de contenido y predictiva del modelo de formulación derivado del análisis preliminar, teniendo en cuenta que solo a través de este proceso es posible establecer un modelo preciso y generalizable a una amplia población atendida por terapeutas que pueden tener diferentes características (Carrascoso \& Valdivia, 2007), incrementando la posibilidad de derivar predicciones eficaces, efectivas y eficientes (Ferro \& Vives, 2004), que mejoren la calidad de vida de los consultante, y la práctica profesional (Carrascoso \& Valdivia, 2007).

\section{Método}

\section{Diseño}

Estudio con metodología mixta que integra niveles de análisis descriptivos y experimentales de caso único A-B-con seguimiento.

\section{Participantes}

En la etapa inicial de la investigación, cuatro estudiantes de pregrado de Psicología y una estudiante de Maestría revisaron 222 historias clínicas, llevadas a cabo por terapeutas en el enfoque comportamental, en un centro universitario de atención psicológica de la ciudad de Bogotá. De manera simultánea, se efectuó la validación de contenido por parte de 19 jueces (entre ellos $\mathrm{PhD}$, magísteres y psicólogos graduados).

En la segunda etapa, participaron una Especialista en Psicología Clínica ComportamentalCognoscitiva (con tres años y seis de experiencia) y una Psicóloga graduada (con dos años y seis de experiencia) que identificaron, clasificaron y calificaron las hipótesis explicativas. Posteriormente, otros dos jueces (una Especialista en Psicología Clínica Comportamental-Cognoscitiva y una psicóloga graduada) analizaron 29 formulaciones clínicas llevadas a cabo con el modelo derivado de las fases previas.

Para la tercera etapa, se escogieron tres casos atendidos por la investigadora, quien era supervisada por otra psicóloga maestra en Psicología con énfasis en Análisis de Comportamiento y candidata a Doctora en Psicología Clínica. Los consultantes fueron dos mujeres y un hombre, que asistieron a un centro universitario de atención psicológica y se seleccionaron por conveniencia, de acuerdo con dos criterios de inclusión: (a) haber sido formulados con el modelo derivado de las primeras fases de la investigación y (b) haber finalizado el proceso, tras cumplir con los objetivos planteados en el plan de tratamiento.

\section{Instrumentos}

Para la validación de contenido del modelo de formulación de caso, se utilizó la modificación del modelo desarrollado por Caycedo et al. (2008) y Pachón y Novoa-Gómez (2009), cuyos cambios se hicieron con base en el acuerdo interjueces y las categorías derivadas del CFCCM.

Además se hizo una base de datos, en la que se incluía la información demográfica de los consul- 
tantes y los datos del proceso terapéutico, incluyendo dentro de estos las transcripciones de las hipótesis funcionales.

Para determinar la confiabilidad de las categorías explicativas utilizadas en las hipótesis funcionales, se usó un formato de acuerdo entre jueces, en el que se incluían estos elementos, y allí debían señalar si en ellas se utilizaban las categorías mencionadas y el tipo de aspectos que hacían parte de ellas. Este mismo formato se utilizó para el análisis de la confiabilidad del modelo de formulación final.

Por último, para la validación predictiva, se hicieron grabaciones en video de las sesiones terapéuticas y como estrategias de evaluación se utilizaron la entrevista semiestructurada y registro de las Conductas Clínicamente Relevantes (CCR). Durante la fase de seguimiento, los consultantes enviaron registros anecdóticos en los que reportaba el estado tanto de sus comportamientos problemáticos como de sus conductas objetivo.

\section{Procedimiento}

Esta investigación se desarrolló en tres etapas.

Primera etapa. En la etapa inicial, se analizó el formato de formulación derivado de la investigación de Pachón y Novoa-Gómez (2009) por parte de los jueces expertos, en tres momentos: reuniones, sesiones de supervisión y seminarios de caso. En las reuniones se discutían la pertinencia de los elementos utilizados en el modelo de formulación para explicar los casos y la importancia de agregar o eliminar elementos de éste. En las supervisiones y en los seminarios de caso se evaluaba la utilidad de las categorías seleccionadas para la comprensión y explicación de los casos, determinando cuáles de ellas debían ser excluidas o qué elementos no eran contemplados y resultaban pertinentes para el análisis, además de señalar las ventajas y desventajas de éste. Todo ello con el fin de establecer la validez de contenido del modelo.

Simultáneamente, se revisaron y seleccionaron las historias clínicas archivadas entre el año 2005 y el 2008, en un centro universitario de atención psicológica de casos atendidos por terapeutas den- tro del modelo comportamental. Tras este proceso, se identificaron 222 historias clínicas y se descartaron 161, dado que no contenían dentro de sus registros hipótesis explicativas, ni un formato de formulación, derivándose, finalmente, 61 historias que cumplían con los criterios de inclusión para él análisis de datos.

Segunda etapa. En esta etapa se realizó el entrenamiento a dos psicólogas en el modelo CFCCM y se codificaron las historias clínicas seleccionadas, de acuerdo con las categorías propuestas por ellos, con el fin de establecer la confiabilidad de contenido de los descriptores del problema y las categorías explicativas utilizadas en las hipótesis funcionales de las historias clínicas seleccionadas, de acuerdo con su juicio. Así pues, se entregó a cada uno de ellas el formato de evaluación que contenía las hipótesis explicativas y los espacios para calificar los diferentes elementos incluidos en ellas. También se les dio un instructivo en el que se explicaban los elementos por evaluar, los criterios de calificación y el tipo de evaluación que se llevaría a cabo.

De forma concurrente, se llevó a cabo el análisis cualitativo de las narrativas o verbatims de las hipótesis funcionales, los cuales permitieron el ordenamiento conceptual que implicaba la organización de los datos en categorías, con el fin de establecer un sistema de clasificación de acuerdo con las propiedades determinadas en el marco conceptual de la investigación (Strauss \& Corbin, 2002). Siguiendo lo anterior, se utilizó la herramienta de análisis cualitativo Atlas ti 5.0 para hacer el ordenamiento conceptual de las hipótesis explicativas según las categorías propuestas por Caycedo et al. (2008), Eells et al. (1998), y Pachón y Novoa-Gómez (2009), permitiendo observar la forma en que los elementos de unas y otras se tenían en cuenta para describir y explicar los casos.

Posteriormente, se elaboraron redes conceptuales (redes estructurales), que son representaciones gráficas del ordenamiento conceptual y que permitieron visualizar tanto los componentes de la clasificación como las relaciones establecidas entre ellos. Sumado a esto, se estableció la frecuencia con que eran usadas las categorías y se analizó la forma en que se usaban los conceptos dentro de ellas. 
Por último, se estableció el formato final teniendo en cuenta las modificaciones de los modelos previos y los resultados de los análisis de las narrativas. Este fue utilizado por 29 terapeutas y las hipótesis derivadas fueron evaluadas por dos jueces expertos, quienes calificaron, en términos de presencia o ausencia, cada una de las categorías, con el fin de establecer la fiabilidad del modelo.

Tercera etapa. Durante ésta se utilizó el modelo de formulación clínica con tres casos atendidos por una de las autoras de este trabajo. Para cada uno de los casos, se realizó una evaluación continua y sistemática, teniendo en cuenta los elementos mencionados en el modelo. La duración de esta etapa fue entre cuatro y ocho sesiones en total, para los casos estudiados, los cuales fueron atendidos en una cámara de Gesell con el fin de grabar en videocintas cada una de las sesiones que fueron vistas por la investigadora y la supervisora, con autorización de los consultantes, quienes firmaron un consentimiento informado, en el que se estipulaban los términos de uso del material, la confidencialidad de sus datos de identificación y se daba autorización para grabar las sesiones con fines terapéuticos e investigativos. Posteriormente, se llevó a cabo la formulación de los tres casos y teniendo en cuenta los elementos explicativos que se derivaron de ésta, se establecieron los objetivos terapéuticos y el plan de intervención para cada uno de los casos. Todo esto permitió establecer la eficacia de la intervención y la validez predictiva del modelo.

\section{Análisis de los datos}

El análisis cuantitativo de las historias clínicas se llevó a cabo a través del programa estadístico SPSS 17.0, por medio del cual se estableció la confiabilidad del contenido de las categorías explicativas, a través del acuerdo entre jueces con el coeficiente Kappa de Cohen y el índice de concordancia entre jueces.

Con respecto al análisis cualitativo de los datos se utilizó el programa ATLAS ti 5.0, con el fin de explorar las categorías explicativas utilizadas en el modelo comportamental-cognoscitivo, por medio del estudio de las hipótesis funcionales contenidas en las historias clínicas.

\section{Resultados}

Los resultados se presentan en tres apartados. Inicialmente, se expone el análisis cualitativo de las hipótesis contenidas en las historias clínicas, a través de las cuales se derivaron categorías explicativas y se fundamentaron las otras categorías establecidas en el CFCCM; posteriormente, se presentan los análisis de la confiabilidad de las formulaciones previas y del modelo actual y, por último, se muestran los resultados de la validez predictiva del mismo.

\section{Análisis cualitativo}

El análisis cualitativo se llevó a cabo con los verbatims de las hipótesis funcionales incluidas en las 61 historias clínicas seleccionadas. Los resultados son presentados en gráficos de frecuencias y también en redes estructurales, que muestran la forma en que se relacionan las narrativas. Cabe señalar que el análisis cualitativo se efectuó con base en los supuestos de la teoría fundamentada y los datos que se registran en las redes, hacen alusión a la fundamentación y a la densidad de las categorías allí presentadas. La fundamentación hace referencia al número de citas que se codifican en una misma categoría, permitiendo ver la validez y solidez de ésta, mientras la densidad muestra la multiplicidad de relaciones existentes entre la categoría con otras. Estos dos elementos se encuentran contenidos en las redes en la parte superior de los cuadros y son presentados entre corchetes, de la siguiente forma: $\{1-3\}$ donde la primera cifra hace referencia a la fundamentación y la segunda a la densidad.

Inicialmente, se realizó la organización conceptual de los contenidos de las hipótesis funcionales de acuerdo con las cinco categorías centrales de análisis ${ }^{1}$, éstas son: (a) descripción del proble-

1 Utilizadas en la formulación de los casos clínicos desde la perspectiva comportamental, derivadas de las investigaciones de Eells, Kendjelic y Lucas (1998), Caycedo, Ballesteros y Novoa (2008), y Pachón y Novoa-Gómez (2009). 
ma, (b) factores de predisposición, (c) factores de adquisición, (d) factores desencadenantes y (e) mecanismos inferidos - que desde el modelo comportamental hacen referencia a los factores de mantenimiento- (Graña, 2005).

La Figura 1, muestra las categorías explicativas que dan cuenta de la función del problema en los cuatro elementos utilizados y la forma en que ellos se agrupan en la misma familia, ésta congrega los componentes principales. También presenta la fundamentación y la densidad de cada una de ellas, evidenciándose que los factores predisponentes es la categoría que tiene más subcategorías asociadas, contrario a los factores de adquisición cuya densidad es la menor en comparación con las otras.

En todas las hipótesis, se hizo una descripción del problema; sin embargo, no en todos los casos se mencionaban de forma simultánea las cuatro categorías explicativas, para dar cuenta de las dificultades. Al respecto, se observó que generalmente los clínicos hacían una descripción de los mecanismos inferidos con mayor frecuencia en comparación con las otras categorías explicativas, mencionando en ocasiones de manera concurrente más de un mecanismo para el mismo consultante. En contraposición, la categoría de los factores desencadenantes/precipitantes fue la que menos se mencionó (Figura 2).

Tras el análisis de los diferentes elementos que conformaban las categorías explicativas y la descripción del problema, se encontró que los pensamientos disfuncionales, el seguimiento de reglas que gobiernan la conducta y los aspectos problemáticos de otras personas, eran subcategorías compartidas por más de uno de estos elementos. Estas tres subcategorías fueron mencionadas por los terapeutas dentro de los mecanismos explicativos de las problemáticas y sumado a ello fueron con-

Figura 1

Categorías explicativas utilizadas por los clínicos para la comprensión de los casos

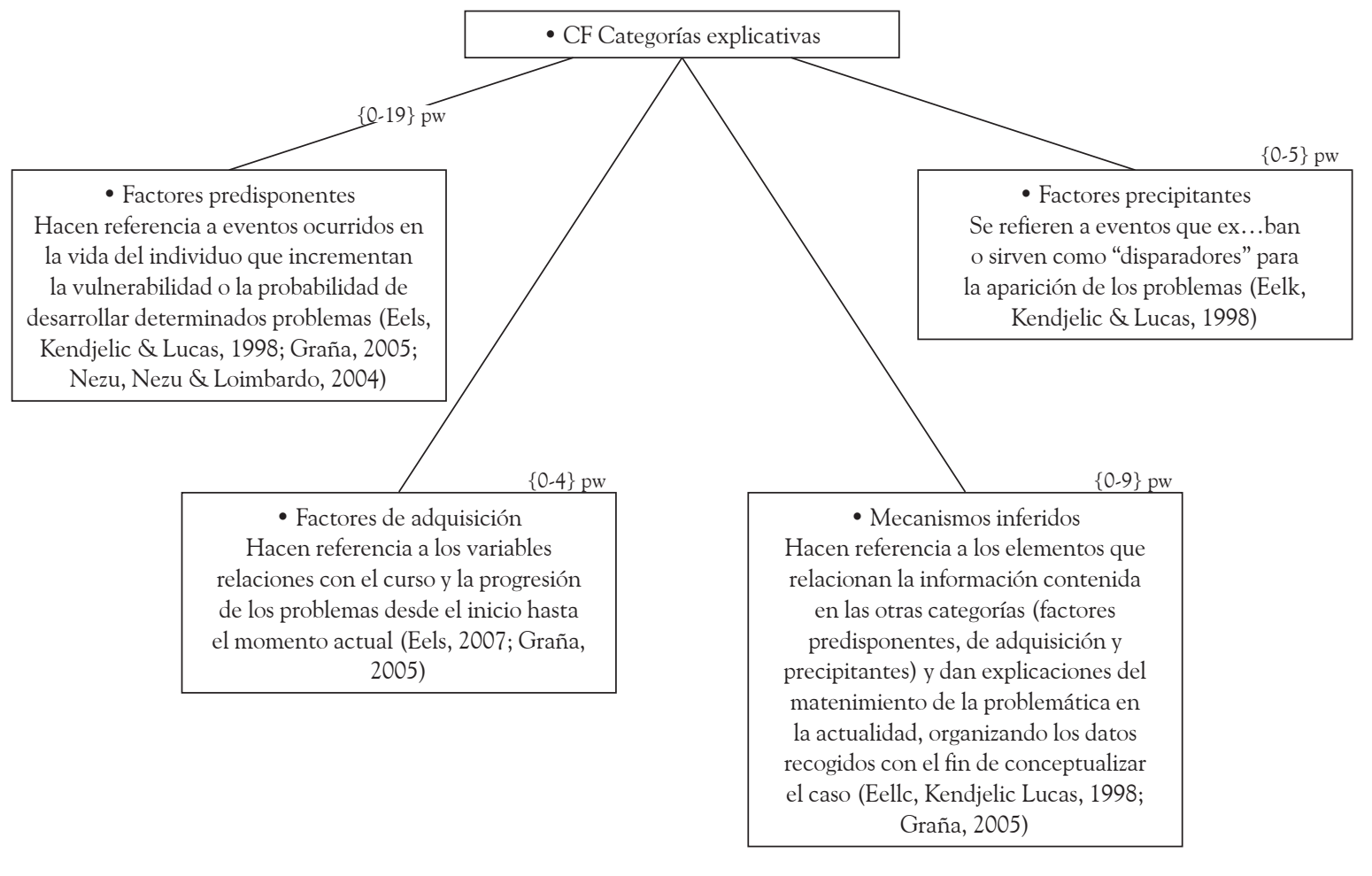

Fuente: elaboración propia. 
FIGURA 2

Frecuencia con la que se mencionan los elementos que constituyen las categorías explicativas

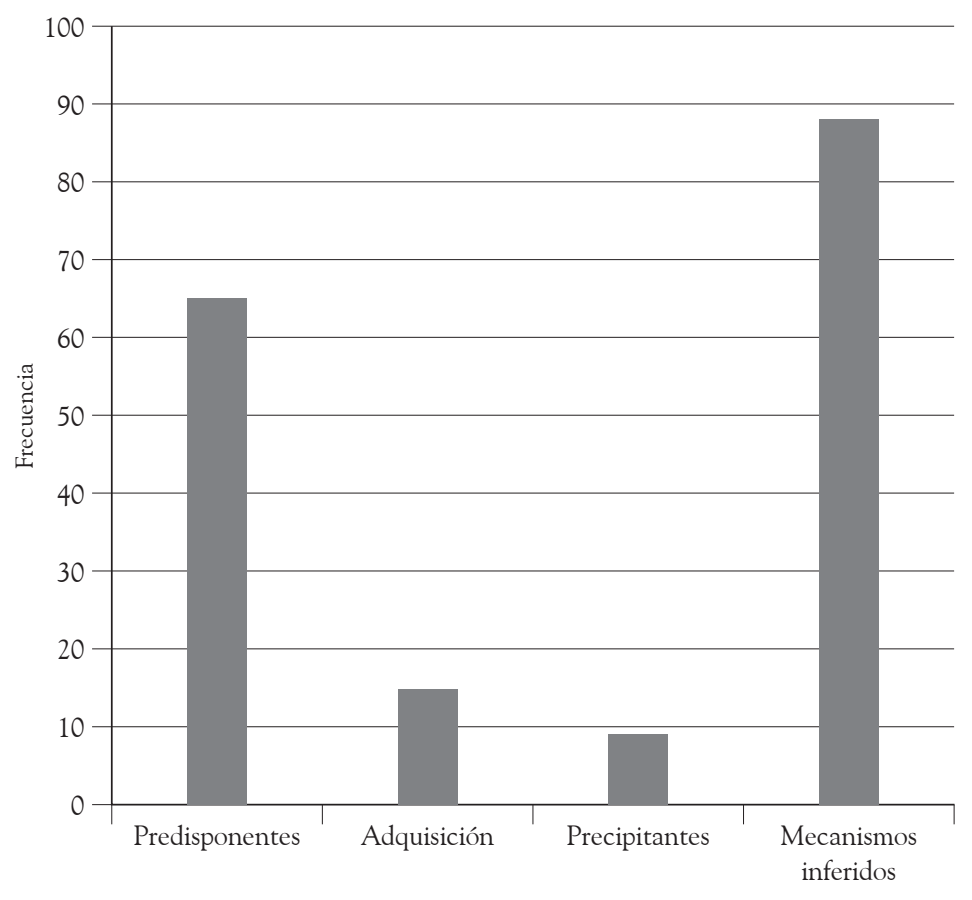

Categorías explicativas

Fuente: elaboración propia.

templadas en otras dos categorías: como factores de predisposición, en el caso de los pensamientos disfuncionales y los aspectos problemáticos de otras personas, además de señalarse como descriptores de la problemática en el caso del seguimiento de reglas que gobiernan la conducta y los pensamientos disfuncionales.

Los datos muestran que los factores predisponentes (Figura 1) son aquellos que presentan una mayor fundamentación, donde los aspectos problemáticos de otras personas, los problemas en los estados afectivos y emocionales de las personas con las que interactúa, las relaciones problemáticas entre los padres, el consumo de alcohol de la pareja, las dificultades para establecer reglas y normas, etc., fueron señalados con mayor frecuencia, diferenciándose de los otros elementos de manera importante, pues, por un lado, los factores precipitantes solo tienen cuatro elementos asociados, y los factores de adquisición tres, evidenciando una menor diversidad de componentes.
Asimismo, la Figura 3 muestra la densidad y la fundamentación de los componentes de los factores precipitantes; de acuerdo con ello, los terapeutas contemplan en sus análisis cuatro tipos de factores desencadenantes los cuales parecen estar relacionados como momentos en la vida de los consultantes en los que hay un cambio importante en la regularidad de su vida cotidiana.

Con respecto a los factores de adquisición (Figura 4), los terapeutas hacían uso de tres tipos de elementos dentro de esta categoría: modelamiento, instrucciones y castigo de repertorios funcionales, éste último se asoció con tres de los quince verbatims de las hipótesis funcionales incluidos en las historias clínicas que fundamentaban los factores de adquisición.

En cuanto a los mecanismos inferidos (Figura 5), se observó que los terapeutas utilizaban al menos siete tipos de elementos para explicar el mantenimiento de la problemática. El mecanismo que tenía una mayor densidad fue el análisis 
FigurA 3

Elementos que constituyen los factores desencadenantes

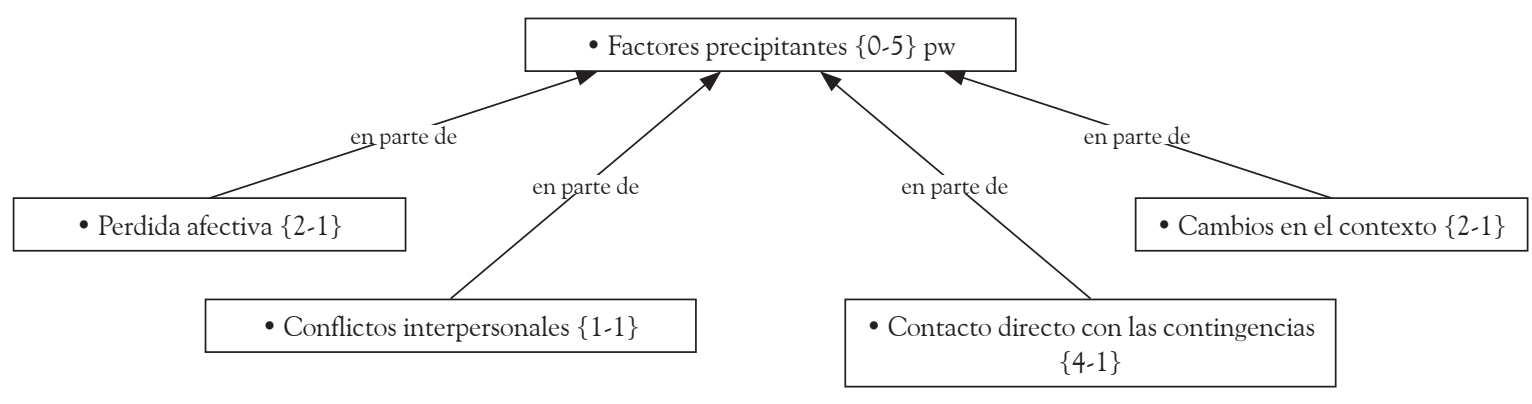

Fuente: elaboración propia.

de contingencias $\{0-6\}$ y sus subcategorías fueron las que contenían la mayor fundamentación. En contraposición, la ausencia de contingencias adecuadas y la búsqueda de control sobre el contexto por parte del consultante fueron las subcategorías con un menor peso $\{1-1\}$.

Figura 4

Elementos que constituyen los factores de adquisición
En relación con la subcategoría de análisis de contingencias, se observó que en ocasiones los terapeutas mencionaban de forma concurrente más de uno de los elementos enmarcados en ella, para explicar las problemáticas de los consultantes. Además, se encontró que algunas de las hipótesis

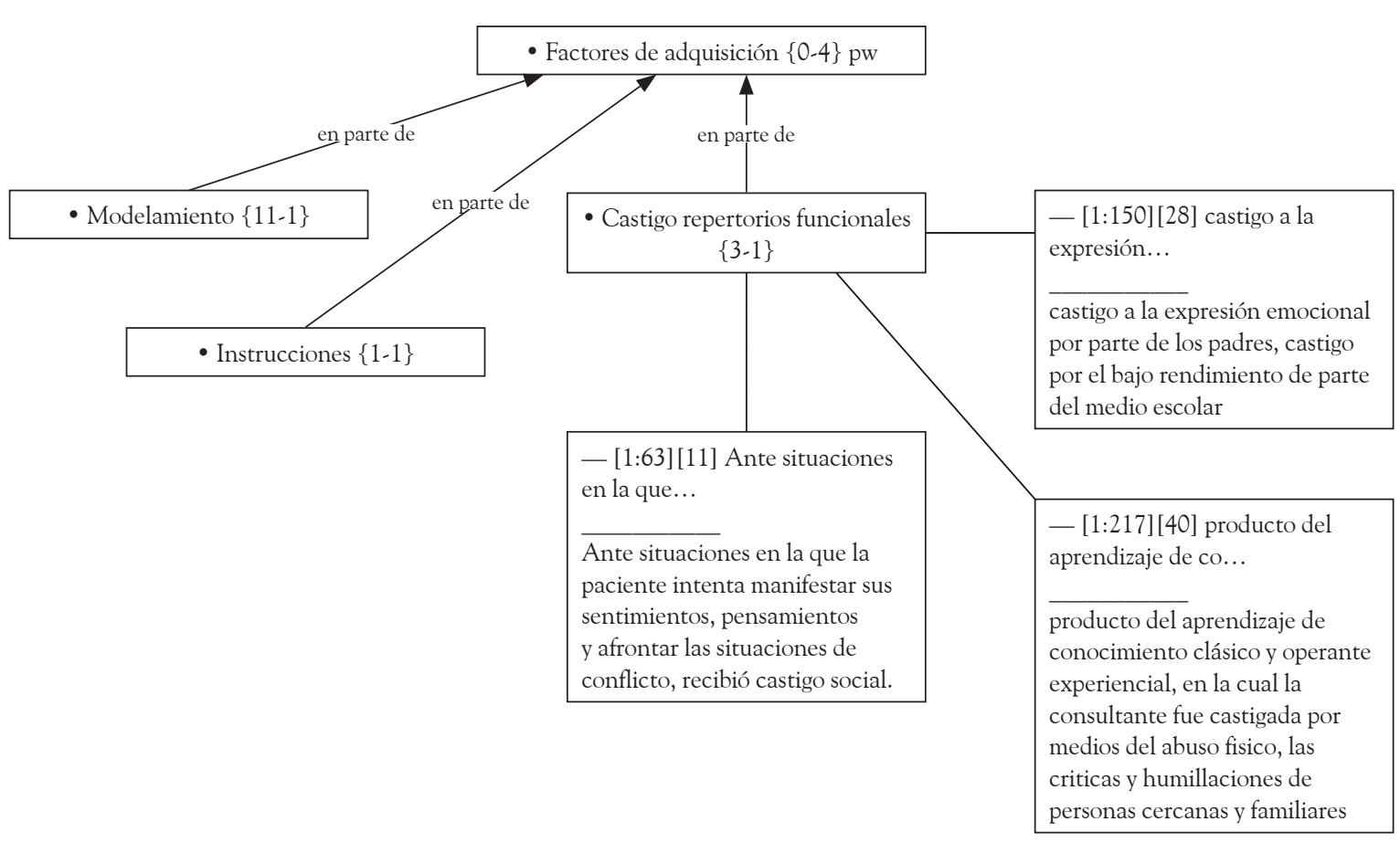

Fuente: elaboración propia. 
explicativas tenían una descripción detallada en la que se incluía no solo el procedimiento de reforzamiento, sino también el tipo de programa en el que éste era administrado y las características del mismo. También se evidenció que algunas hipótesis tenían dificultades para utilizar un lenguaje adecuado en el momento de identificar los mecanismos involucrados en el mantenimiento, haciendo uso de lenguaje ajeno a la disciplina.

Estos análisis permitieron ver que, en efecto, los terapeutas dentro del modelo conductual hacían uso de las categorías de descripción del problema, predisponentes, precipitantes adquisición y mantenimiento, en el momento de abordar las problemáticas y explicarlas, aunque el uso que se les daba a cada una de ellas variaba en frecuencia y calidad de la información que se consignaba en ellos.

\section{Análisis de confiabilidad de las categorías explicativas utilizadas en las historias clínicas}

Siguiendo la metodología del CFCCM se llevó a cabo el análisis de la confiabilidad (Tabla 1). Los resultados mostraron que dos de las cinco categorías fueron moderadamente confiables en las historias clínicas revisadas, los predisponentes, cuyo índice Kappa fue de 0.50 y los mecanismos inferidos, con un coeficiente de 0.46 . La categoría referente a la descripción del problema no pudo ser sometida a los análisis de confiabilidad con el coeficiente Kappa, dado que para uno de los jueces ésta estuvo presente en todos los casos, por lo que era una constante y al no haber variabilidad no era posible determinarlo por medio de dicho análisis.

FIGURA 5

Fundamentación y densidad de los mecanismo inferidos

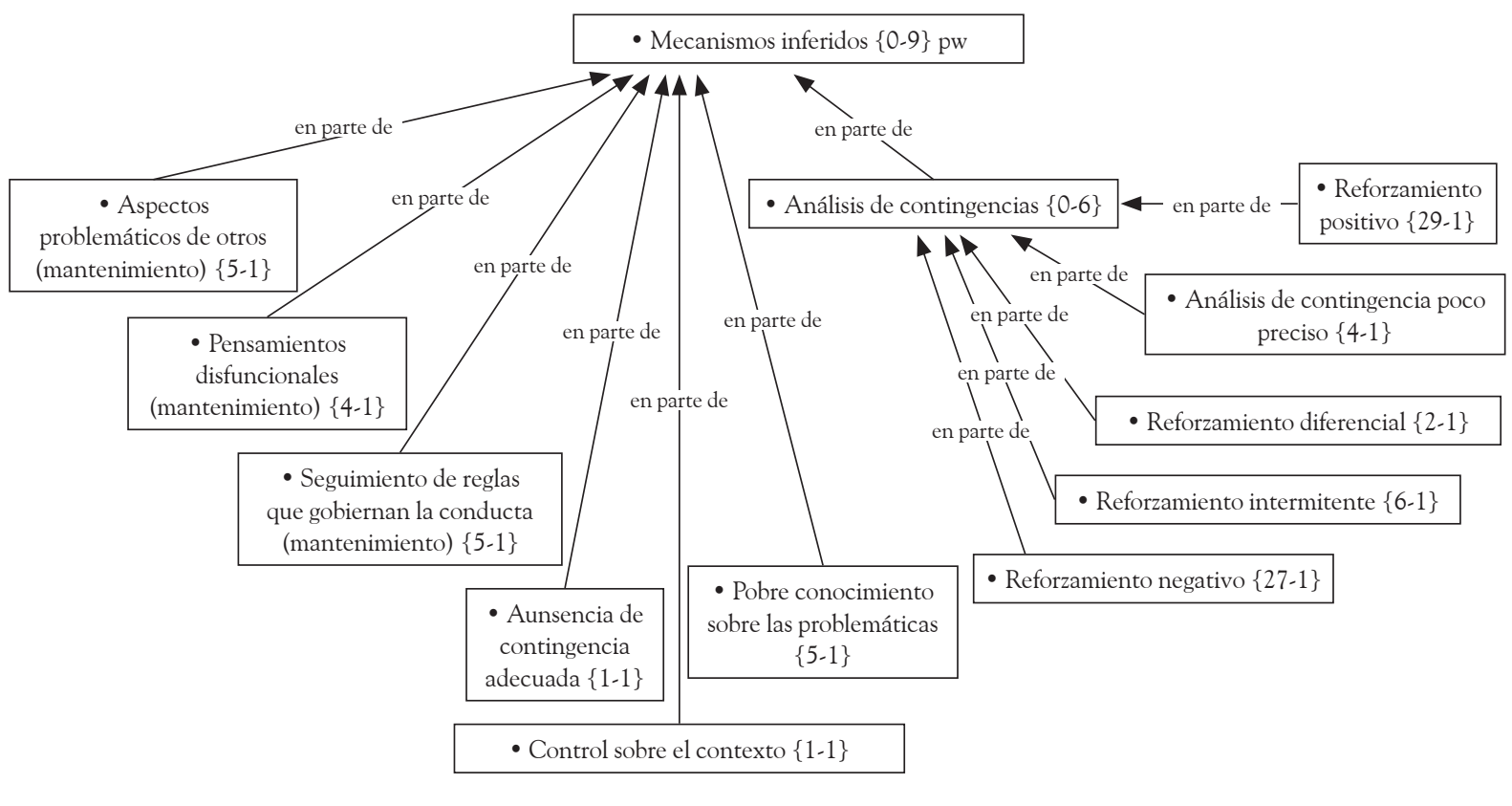

Fuente: elaboración propia. 
TABLA 1

Análisis de la confiabilidad de las categorías descriptivas y explicativas a través del Coeficiente Kappa de Cohen

\begin{tabular}{lcc}
\hline \multicolumn{1}{c}{$\begin{array}{c}\text { Elementos de las categorías } \\
\text { en la formulación }\end{array}$} & Kappa & Significación \\
\hline $\begin{array}{l}\text { Descripción del problema } \\
\text { Factores de predisposición }\end{array}$ & 0.50 & -0.000 \\
Factores precipitantes & 0.23 & 0.051 \\
$\begin{array}{l}\text { Factores de adquisición } \\
\begin{array}{l}\text { Mecanismos inferidos (Factores } \\
\text { de mantenimiento) }\end{array}\end{array}$ & 0.45 & 0.003 \\
\hline
\end{tabular}

Fuente: elaboración propia.

Posteriormente, se evaluó la confiabilidad de las categorías en el modelo de formulación desarrollado en esta investigación, a través del índice de concordancia entre jueces (Tabla 2). Los datos mostraron una fiabilidad casi perfecta en las categorías explicativas, dado que los índices obtenidos fueron superiores a 0.80 , y perfecta para la descripción del problema, pues jueces acordaron que en todos los casos era mencionada.

TABLA 2

Análisis de la confiabilidad de contenido de las categorías descriptivas y explicativas a través del índice de concordancia entre jueces

\begin{tabular}{lc}
\hline $\begin{array}{c}\text { Elementos de las categorías en la } \\
\text { formulación }\end{array}$ & $\begin{array}{c}\text { Índice de } \\
\text { concordancia }\end{array}$ \\
\hline Descripción del problema & 1.00 \\
Factores de predisposición & 0.86 \\
Factores precipitantes & 0.86 \\
Factores de adquisición & 0.82 \\
$\begin{array}{l}\text { Mecanismos inferidos (factores de } \\
\text { mantenimiento) }\end{array}$ & 0.86 \\
\hline
\end{tabular}

Fuente: elaboración propia.
Además, se estableció el coeficiente Kappa, con el fin de seguir la metodología utilizada en el primer análisis y en el CFCCM. Este análisis no permitió establecer la confiabilidad de la descripción del problema, dado que ésta fue constante para los dos jueces y, aunque se encontró que las categorías de factores precipitantes y de adquisición fueron moderadamente confiables, los predisponentes y de mantenimiento no fueron confiables y tampoco fueron significativos, por lo que estos datos no resultan validos (Ato, Benavente \& López, 2006).

\section{Validación del modelo de formulación (validez predictiva)}

Para determinar este tipo de validez, se llevaron a cabo tres estudios de caso único que fueron formulados con el modelo desarrollado en el presente estudio, haciendo uso de las categorías explicitadas a lo largo del documento y cuya intervención fue realizada con base en las hipótesis derivadas de ese análisis. En los tres casos se midieron las Conductas Clínicamente Relevantes (CCR) y Problemas en la Vida Diaria (MVD) y Mejorías en la Vida Diaria (MVD).

En la Figura 5, se pueden observar los cambios en el comportamiento del caso 1 tras introducir la intervención derivada de la formulación clínica, de tal forma que las CCR1 (problemas en consulta) disminuyeron su frecuencia de forma importante en dicha fase, mientras que las CCR2 (mejorías en consulta) tuvieron el efecto contrario incrementando su presentación. Cabe señalar que las sesiones 10 y 11 hacen referencia al seguimiento que se hizo un mes y tres meses, respectivamente, después de finalizar el tratamiento y permiten ver el mantenimiento de las mejorías.

De forma similar, la Figura 6 muestra los cambios en las CCR del caso 2 y se observan cambios importantes a partir de la introducción del tratamiento, aunque la Figura 7 presenta las mejorías en su contexto natural y permite conocer como se mantienen estos tras finalizar la intervención, después de dos y tres meses, que se presentan como las sesiones 15 y 16 . 
FigURA 5

Frecuencia con la que el consultante en el caso 1 reportó CCR1, CCR2, Problemas en la Vida Diaria PVD y MVD

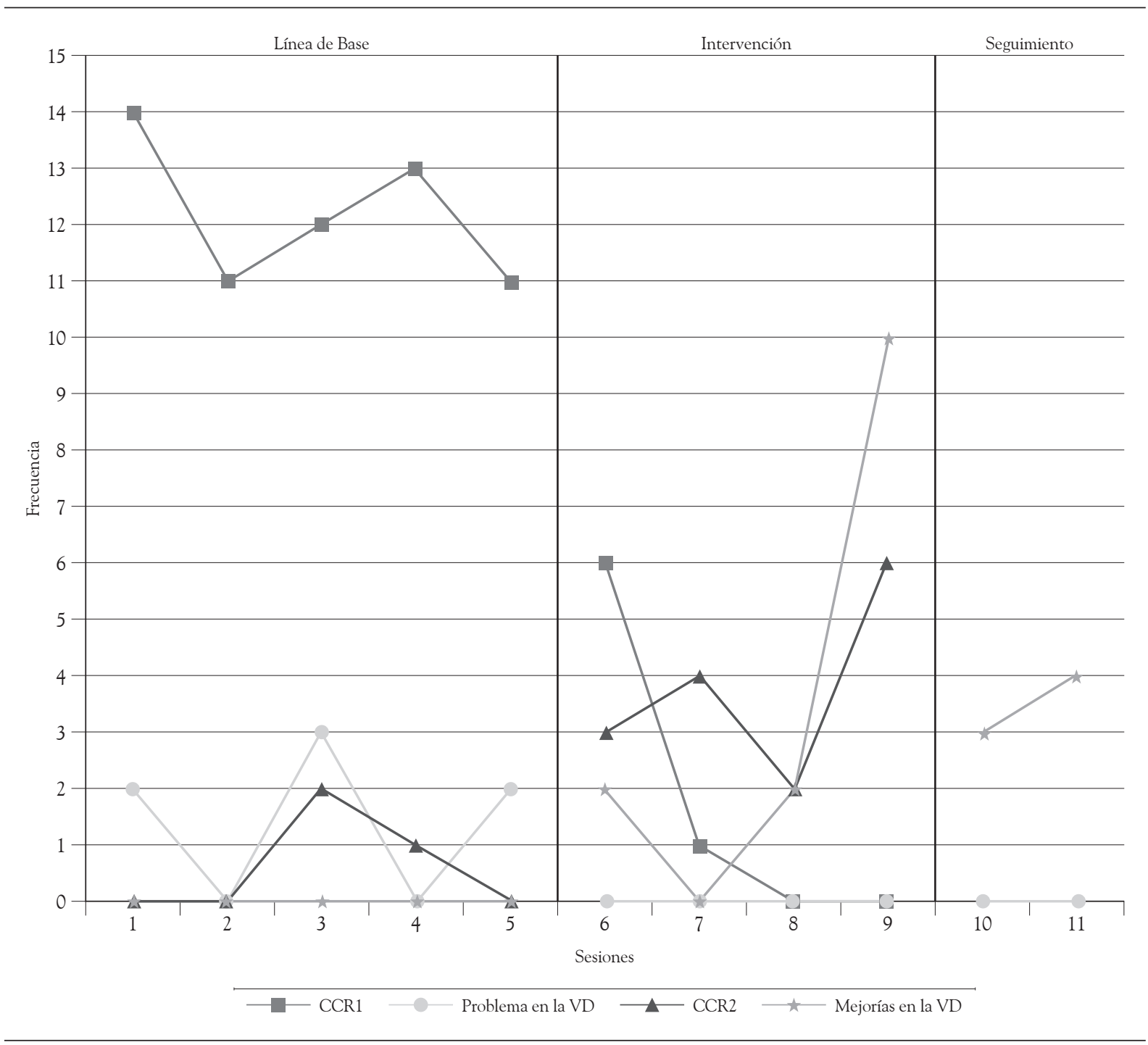

Fuente: elaboración propia. 
FIGURA 6

Frecuencia con la que el consultante en el caso 2 refiere las CCR1 y las CCR2

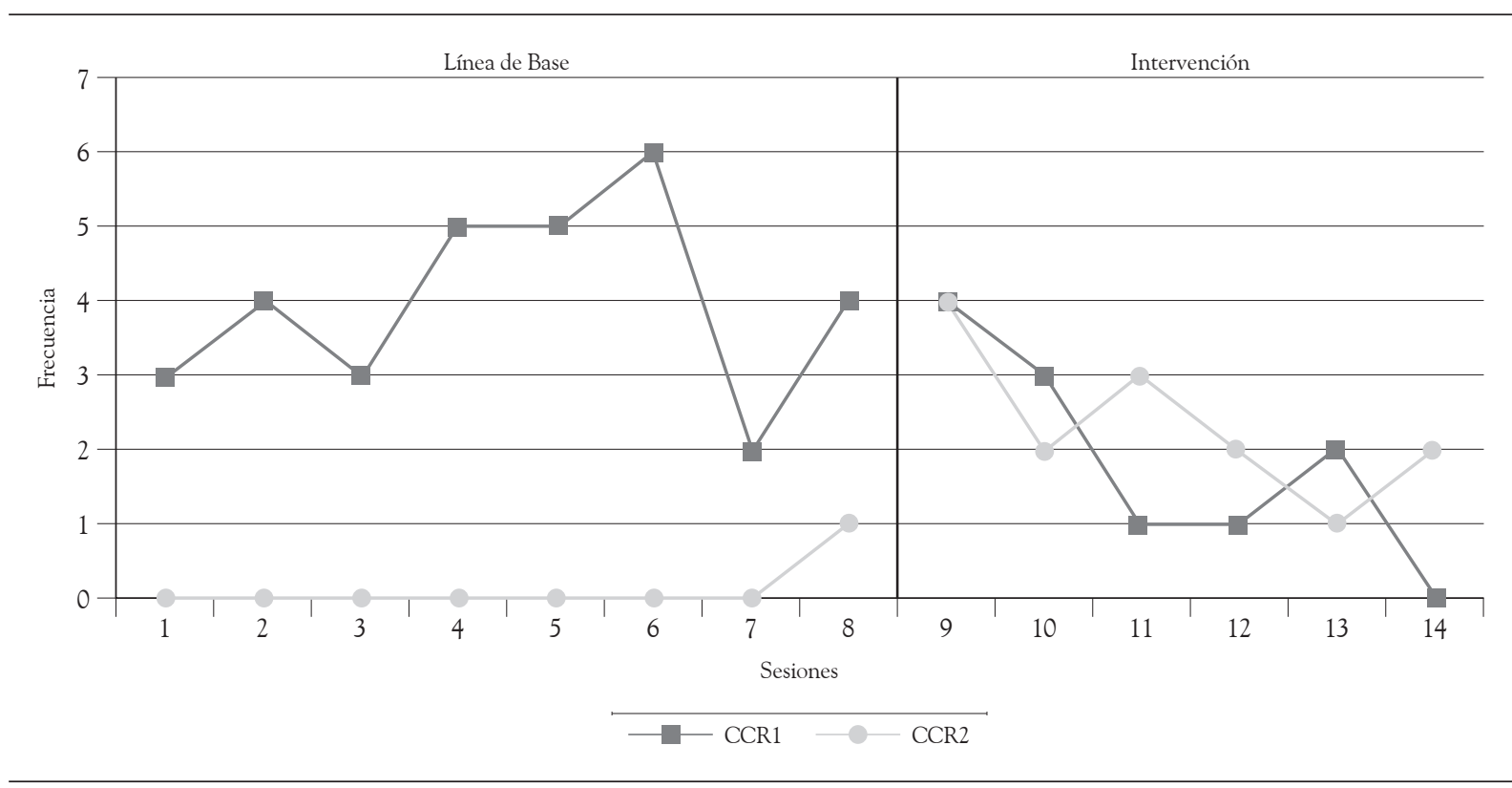

Fuente: elaboración propia.

FIGURA 7

Frecuencia con la que el consultante en el caso 2 reportó MVD

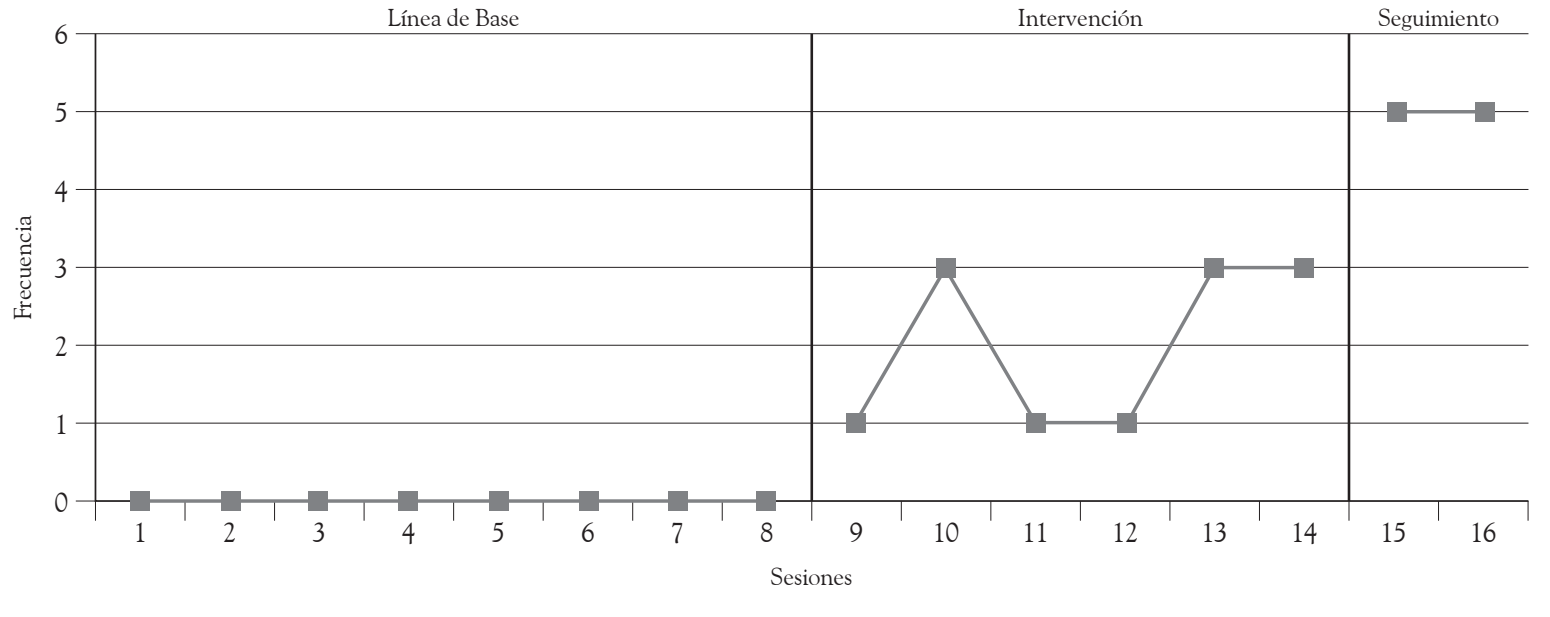

Fuente: elaboración propia. 
Por último, la Figura 8 permite ver los cambios en las CCR del caso 3, tras la introducción de la intervención diseñada con base en las hipótesis establecidas, a partir de la organización de la información en el modelo de formulación. Además, la Figura 9 muestra los cambios y mejorías del caso, en los contextos ajenos al de consulta observándose también cambios ellos.

En general, los resultados del presente estudio brindan información de distinto orden para el lector que se encuentra relacionada con la metodología de formulación de caso, desde la perspectiva conductual, mostrando los elementos que se utilizan con mayor frecuencia desde esta postura para dar cuenta de los casos. Además, se enseñan datos cualitativos y cuantitativos relacionados con las categorías (factores de predisposición, factores precipitantes, factores de adquisición y factores de mantenimiento) usadas en el modelo para explicar los casos, mostrando la densidad, la fundamentación, la confiabilidad y la validez de éstas. En el siguiente apartado se discuten estos resultados.

\section{Discusión}

Los resultados mostraron confiabilidad de las categorías explicativas relacionadas con los factores predisponentes y los mecanismos inferidos (factores de mantenimiento) en las hipótesis y formulaciones de las historias clínicas analizadas

Figura 8

Frecuencia con la que el consultante en el caso 3 refiere las CCR1 y las CCR2

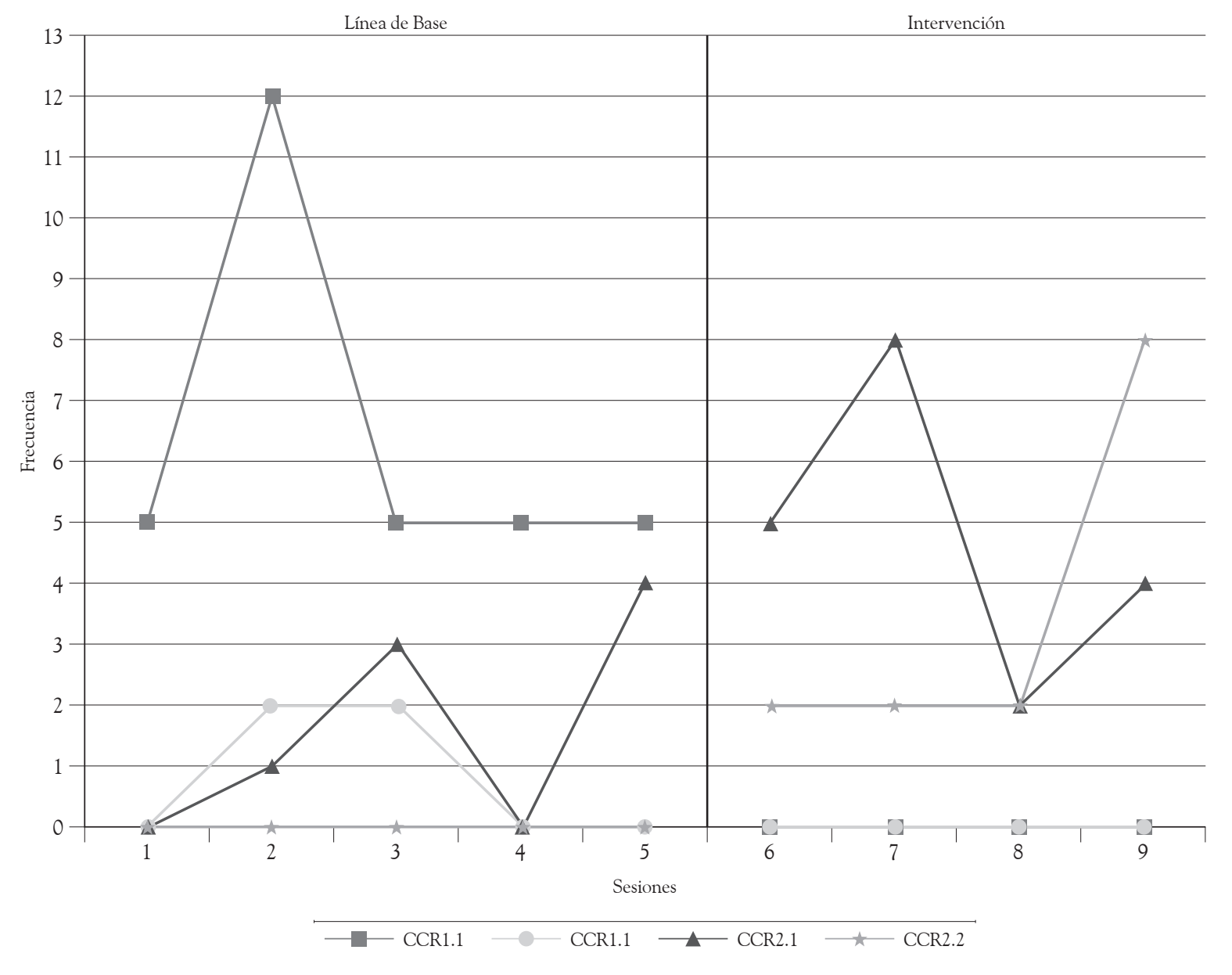

Fuente: elaboración propia. 
Figura 9

Frecuencia con la que el consultante en el caso 3 refiere PVD y MVD

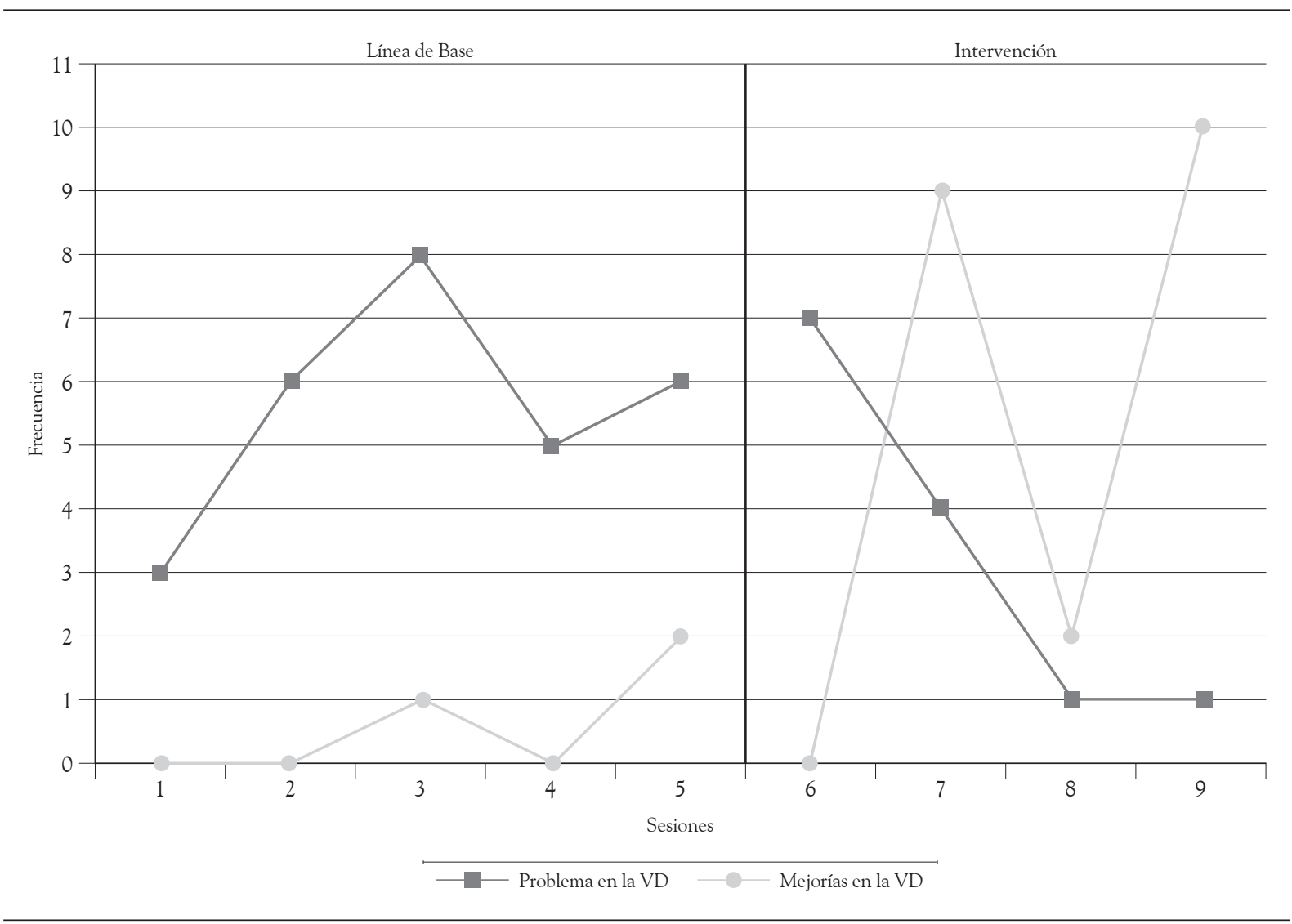

Fuente: elaboración propia.

de los años 2005 al 2008, en contraposición con los factores precipitantes y de adquisición que no mostraron confiabilidad. En cuanto a la categoría de descripción del problema no fue posible establecer el Coeficiente Kappa de Cohen, dado que para uno de los jueces éste fue un dato constante. $\mathrm{Al}$ respecto cabe señalar que a través del análisis cualitativo que se llevó a cabo con estos datos, se observó que las hipótesis de los factores precipitantes y de adquisición fueron mencionadas con poca frecuencia en las historias clínicas y los jueces estuvieron de acuerdo en que no se hacía mención de ellas; es posible que debido a esto no fueran confiables.

En relación con lo expuesto, es factible que uno de los problemas que hay en la identificación de los factores precipitantes y de adquisición por parte de los terapeutas, sea la relación que guar- dan, por un lado, los factores precipitantes con la descripción de momentos específicos en la historia del individuo (Eells et al., 1998) y, por otro lado, la dependencia que tienen los factores de adquisición con la identificación de los procesos de aprendizaje que estuvieron involucrados en el desarrollo y evolución de la problemática hasta consolidarse dentro del repertorio conductual, lo que puede ser difícil de describir para muchas de las personas que acuden a consulta (Eells, 2007; Graña, 2005), además de las dificultades que durante años han tenido los psicólogos conductuales para definir y diferenciar estos últimos de otros factores, pasando por alto los datos que se tienen de este factor dentro de la investigación básica en Psicología, haciendo evidente la falencia que señalan Carrascoso y Valdivia (2007), en relación con los problemas que tienen los psicólogos para integrar los datos de este tipo 
de investigación con la Psicología aplicada, que, de acuerdo con ellos, disminuye la oportunidad para validar las categorías explicativas dentro del modelo conductual.

Las dificultades encontradas en los factores de adquisición y precipitantes llevan a pensar si la función planteada hasta el momento por los diferentes autores (Eells, 2007; Eells et al., 1998; Graña, 2005) con respecto a los elementos asociados con la adquisición e inicio de las problemáticas, ha sido correcta, pues si bien la identificación de estos factores le permite al psicoterapeuta establecer el grado de afectación, resistencia al cambio, evolución e impacto de la problemática para la persona que consulta, el rol que juegan para la conceptualización del caso parece estar relacionado con aspectos descriptivos y no explicativos, pues contrario a lo observado en las historias clínicas con respecto a los factores de predisposición y mantenimiento, aparentemente, la identificación de la función de la conducta no dependen de esos dos factores. Sin embargo, se plantea que es necesario investigar con mayor profundidad al respecto, y establecer si los problemas se relacionan con la conceptualización o con la utilidad de los factores de adquisición y precipitantes, para la explicación de la conducta.

Con respecto a los factores de predisposición y mantenimiento, los resultados permitieron ver que resultaron confiables, a pesar de contener dentro de sus subcategorías elementos que pertenecían a diferentes posturas dentro de la perspectiva conductual. Esto tiene una implicación importante para el modelo de formulación desarrollado en esta investigación, pues permite ver que éste no se encuentra adscrito, de manera formal, a ninguna corriente, ya que dentro de sus mecanismos de mantenimiento los psicoterapeutas pueden incluir tanto elementos relacionados con posturas mediacionales como no mediacionales, ampliando la posibilidad de hacer uso de él, no sólo en el contexto terapéutico sino también investigativo, por la flexibilidad que muestra para adaptarse a los diferentes marcos conceptuales dentro del paradigma conductual.

A partir de ello, el modelo de formulación diseñado en el presente estudio usó dichas categorías para explicar los casos y señaló de forma explícita la importancia de identificarlas, para dar cuenta del comportamiento problema. Para establecer su confiabilidad, se utilizaron el coeficiente Kappa y el índice de concordancia entre jueces; el primero de ellos resultó problemático dado que las evaluaciones hechas por los jueces tenían un efecto de prevalencia, donde se pudo observar una proporción global extrema en el análisis de las categorías y, como se ha encontrado en múltiples investigaciones, esto invalida el uso de este estadístico (Agresti, Ghosh \& Bini, 1995; Ato et al., 2006; Byrt, Bishop \& Carlin, 1993; Feinstein \& Cicchetti, 1990; Hoehler, 2000; Lantz \& Nebenzahl, 1996; Spitznagel \& Hazer, 1985). Por lo que el uso del índice de concordancia entre jueces fue la opción óptima para establecer la fiabilidad del modelo con una mayor certeza y, como se pudo observar, mostró que todas las categorías del modelo fueron altamente confiables. Sin embargo, dadas las dificultades con el coeficiente Kappa se sugiere, en futuras investigaciones, realizar análisis que impliquen otros jueces, estableciendo la fiabilidad con modelos loglineales como lo proponen Ato et al. (2006), o incrementando el número de hipótesis a evaluar para ver si ello ayuda a incrementar la variabilidad en las calificaciones, y así poder utilizar el coeficiente Kappa sin problemas.

Con respecto a la validación de contenido del modelo, los jueces determinaron a través del proceso que las categorías utilizadas para describir y explicar los repertorios problemáticos resultaban válidas, siendo éstas útiles y pertinentes para los objetivos planteados. En cuanto a la validez predictiva, se estableció por medio del uso de la formulación en casos clínicos y a través de ellas se derivaron planes de tratamiento que predijeron de forma eficaz los elementos sobre los cuales era pertinente intervenir, para lograr resultados positivos, mostrando así que la formulación fue válida en este aspecto. En relación con ello, hay que señalar que, si bien se logró determinar la validez de contenido y predictiva, es importante determinar la validez concurrente, pues así se podría saber si el modelo permite a diferentes terapeutas con un mismo enfoque teórico establecer hipótesis simi- 
lares (Martínez-García \& Martínez-Caro, 2006; Mumma \& Smith, 2001), y la validez experimental del modelo, dado que sólo a través de ésta es posible determinar si esta es legítima a nivel clínico como lo plantean Carrascoso y Valdivia (2007), por lo que se recomienda que, para futuras investigaciones, se haga un mayor énfasis en este aspecto y se reporten casos que contengan fases de seguimiento que permitan ver si los resultados se mantienen a través del tiempo, después de finalizada la intervención.

A manera de conclusión, se puede decir que, si bien los resultados muestran que el modelo de formulación presentado en la presente investigación mostró ser válido a nivel de contenido y predictivo, es importante determinar la validez concurrente y clínica del mismo debido a que ello permitirá conocer si esta metodología garantiza la elaboración de planes de intervención eficientes, eficaces y efectivos (Carrascoso \& Valdivia, 2007). Además se encontró que las categorías explicativas relacionadas con los factores predisponentes y de mantenimiento resultaron ser confiables, siendo la subcategoría relacionada con el análisis de las contingencias de refuerzo la más confiable para dar cuenta de las problemáticas de los individuos, lo que se relaciona no solo con los datos derivados del análisis cualitativo de la presente investigación, sino también con las bases teóricas y conceptuales del modelo comportamental, por lo que resulta evidente la importancia de incluir un apartado dentro de los mecanismos inferidos que haga énfasis en este aspecto. Sin embargo, es importante ampliar la muestra de formulaciones llevadas a cabo con este modelo, para establecer con mayor certeza la fiabilidad del modelo desarrollado en la presente investigación.

Por último, cabe señalar que la presente investigación ha permitido mejorar y reestructurar de manera importante algunas de las limitaciones señaladas en los estudios de Caycedo et al. (2008), y Pachón y Novoa-Gómez (2009) en relación con las categorías utilizadas en el modelo de formulación, pues en este estudio es posible ver con mayor claridad la relación entre los elemento explicativos y los datos empíricos a través de la aplicación hecha del modelo, y la relación de éstas con las bases conceptuales propias de la perspectiva comportamental. Estos hallazgos permiten ver que el Grupo de Investigación en Psicología y Salud ha mantenido continuidad en la labor investigativa relacionada con el bienestar psicológico y de esta manera muestra el compromiso que tiene con el mejoramiento de la calidad de vida de los consultantes, a través del diseño de metodologías válidas y confiables que mejoren la elaboración de planes de intervención más efectivos. Siguiendo esto, se plantea ampliar la investigación al respecto, trabajando sobre las limitaciones del presente estudio y acogiendo algunas de las sugerencias hechas para perfeccionar el modelo de formulación clínica.

\section{Referencias}

Agresti, A., Ghosh, A. \& Bini, M. (1995). Raking kappa: Describing potential impact of marginal distributions on measure of agreement. Biometrical Journal, 37, 811-820.

Arias, M., Fernández, F. \& Perona, S. (2000). Psicología básica, psicología aplicada y metodología de la investigación: el caso paradigmático del análisis experimental y aplicado del comportamiento. Revista Latinoamericana de Psicología, 32, 211-300.

Ato, M., Benavente, A. \& López, J. (2006). Análisis comparativo de tres enfoques para evaluar el acuerdo entre observadores. Psicothema, 18, 638645.

Bergner, R. (1998). Characteristics of optimal clinical case formulations. American Journal of Psychotherapy, 52, 287-301.

Byrt, T., Bishop, J. \& Carlin, J. B. (1993). Bias, Prevalence and Kappa. Journal of Clinical Epidemiology, 46, 423-429.

Carey, M., Flasher, L., Maisto, S. \& Turkat, I. (1984). The a priori approach to psychological assessment. Professional Psychology: Research and Practice, 15, 515-527.

Carrascoso, F. \& Valdivia, S. (2007). Towards alternative criteria for the validation of psychological treatments. International Journal of Psychology and Psychological Therapy, 7, 347-363. 
Caycedo, C., Ballesteros, B. \& Novoa, M. (2008). Análisis de un protocolo de formulación de caso clínico desde las categorías de bienestar psicológico. Universitas Psychologica, 7 (1), 231-250.

Eells, T. (2001). Update on psychotherapy case formulation research. The Journal of Psychotherapy Practice and Research, 10, 277-281.

Eells, T. (2007). History and current status of psychotherapy case formulation. En T. Eells (Comp.), Psychotherapy case formulation (Vol.2, pp. 3-32). New York: The Guilford Press.

Eells, T., Kendjelic, E. \& Lucas, C. (1998). What's in a case formulation? Development and use of a content coding manual. The Journal of Psychotherapy Practice and Research, 7, 144-156.

Feinstein, A. \& Cichetti, D. (1990). High agreement but low Kappa: I. The problem of two paradoxes. Journal of Clinical Epidemiology, 43, 543-549.

Ferro, R. \& Vives, C. (2004). Un análisis de los conceptos de efectividad, eficacia y eficiencia.Panace@, 5, 97-99.

Graña, J. (2005). Formulación de casos en psicología clínica. En V. E. Caballo (Comp.), Manual para la evaluación clínica de los trastornos psicológicos. Estrategias de evaluación, problemas infantiles y trastornos de ansiedad (pp. 99-120). Madrid: Ediciones Pirámide.

Hayes, S. \& Haas, J. (1988). A reevaluation of the concept of clinical significance: Goals, methods, and methodology. Behavioral Assessment, 10, 189-196.

Haynes, S., Leisen, M. \& Blaine, D. (1997). Design of individualized behavioral treatment programs using functional analytic clinical case models. Psychological Assessment, 9, 334-348.

Haynes, S. \& O'Brien, W. (2000). Principles and practice of behavioral assessment. New York: Kluwer/ Plenum.

Hoehler, F. K. (2000). Bias and prevalence effects on kappa viewed in terms of sensitivity and specificity. Journal of Clinical Epidemiology, 53, 499-503.

Iwata, B. \& Worsdell, A. (2005). Implications of functional analysis methodology for the design of intervention programs. Exceptionality, 13, 25-35.

Kahneman, D. \& Klein, G. (2009). Conditions for intuitive expertise a failure to disagree. American Psychologist, 64, 515-526.
Kendjelic, E. \& Eells, T. (2007). Generic psychotherapy case formulation training improves formulation quality. Psychotherapy: Theory, Research, Practice, Training, 44, 66-77.

Lantz, C. A. \& Nebenzahl, E. (1996). Behavior and interpretation of the statistics: Resolution of the two paradoxes. Journal of Clinical Epidemiology, 49, 431-434.

Martínez-García, J. \& Martínez-Caro, L. (2006). La validez discriminante como criterio de evaluación de escalas: iteoría o estadística? Universitas Psychologica, 8 (1), 27-36.

Mumma, G. \& Mooney, S. (2007). Incremental validity of cognitions in clinical case formulation: An intraindividual test in a case example. Journal of Psychopathology and Behavioral Assessment, 29, 17-28.

Mumma, G. \& Smith, J. (2001) . Cognitive-BehavioralInterpersonal scenarios: Interformulator reliability and convergent validity. Journal of Psychophatology and Behavioral Assessment, 23, 203-221.

Muñoz, A. \& Novoa, M. (2009, agosto). Análisis de las variables relacionadas con el éxito terapéutico y las categorías explicativas utilizadas dentro de la formulación clínica conductual. Poster presentado en el II Congreso Nacional de Psicología COLPSIC y Primer Congreso Internacional de Psicología COLPSIC-ASCOFAPSI, Medellín, Colombia

Muñoz, A. \& Novoa, M. (2010). Análisis de las variables asociadas al éxito terapéutico en intervenciones diseñadas dentro del modelo conductual. Pontificia Universidad Javeriana. Documento inédito.

Nezu, A., Nezu, C. \& Lombardo, E. (2004). Cognitive case-formulation and treatment design. A problem solving approach. New York: Springer Publishing Company, Inc.

Pachón, G. \& Novoa-Gómez, M. (2009). Validación de un protocolo de formulación de caso clínico desde la perspectiva del análisis del comportamiento. Tesis para acceder al título de psicólogo, Pontificia Universidad Javeriana, Bogotá.

Rodríguez, M. \& Vanegas, F. (2010). Validación de constructo de la formulación clínica por procesos de Castro y Ángel. Revista Psychologia: Avances de la Disciplina. Manuscrito en preparación. 
Sim, K., Peng, K. \& Bateman, A. (2005). Case formulation in psychotherapy: Revitalizing its usefulness as a clinical tool. Academic Psychiatry, 29, 289-292.

Spitznagel, E. I. \& Helzer, J. E. (1985). A proposed solution to the base rate problem in the kappa statistics. Archives of General Psychiatry, 42, 725-728.

Strauss, A. L. \& Corbin, J. M. (2002). Bases de la investigación cualitativa técnicas y procedimientos para desarrollar la teoría fundamentada. Medellín: Universidad de Antioquia. 
